\title{
2020 SSO Presidential Address: Surgical Oncology Moonshot
}

\author{
David L. Bartlett \\ System Chair, Allegheny Health Network Cancer Institute, Pittsburgh, PA
}

My purpose for this presidential address is to rally the surgical oncology community around moonshot goals that if accomplished will transform what we can do for our patients while preserving the surgical oncologist's role in the multidisciplinary care of patients. As with the mission to reach the moon at a time when the United States was lacking in technology and knowledge, a moonshot goal transforms everything with an aim, a competitive spirit, and ordinary people working together with a passion for success.

So why is it critical to have a moonshot goal? John F. Kennedy announced the moonshot program at my alma mater, Rice University, and rallied the crowd saying, "But why the moon?... Why does Rice play Texas?... not because they are easy, but because that goal will serve to organize and measure the best of our energies and skills" (Fig. 1).

The term "moonshot" has become synonymous with an ambitious, exploratory, and groundbreaking project undertaken with collaboration, creativity, and the mindset for success, and the goal for America was not to gather moon dust. It was far-reaching, about improving all aspects of technology needed to propel the United States into the future. But a defined purpose and timeline rallied the country, the scientists, and the engineers to make many important advances critical to our success as a country. Ultimately, it may have saved the United States from becoming irrelevant on the world stage.

(C) Society of Surgical Oncology 2020

First Received: 8 October 2020

Accepted: 10 October 2020;

Published Online: 24 November 2020

D. L. Bartlett

e-mail: David.Bartlett@ahn.org
In 1957, Sputnik, developed by the Soviet Union, streaked across the sky - the first satellite to orbit the earth. Four years later, the Soviets successfully put the first man in space on Vostok 1. So, when Kennedy announced the moonshot program, we were behind the Russians in technology and space exploration. Kennedy stated: "For while we cannot guarantee that we shall one day be first, we can guarantee that any failure to make this effort will make us last."

Although I would never want to portray that we are in some kind of cold war with the medical oncologists and basic scientists, I would like to use that spirit to awaken the surgical oncologists. We are behind and at risk of being left out.

Although it is not surprising that the 1.8-billion-dollar cancer program was entitled "Cancer Moonshot," it is worth asking, "Where do surgery and surgeons fall into this paradigm?" Although surgery remains the most successful way to cure cancer, surgical science is not represented in any of the funding initiatives, and surgical oncology is not even represented in the blue-ribbon panel that defines the problems to address. The Cancer Moonshot Task Force was created to include seven working groups specific for the largest areas of research in oncology, and not one of them addressed issues specific to surgical oncology. A gap exists between the science and clinical care. Surgical oncology needs its own moonshot. In the same way that the United States was at risk of becoming irrelevant on the world stage if the moonshot program did not bring us back, the field of surgical oncology is at risk if we are not asking the right questions and doing the right trials.

I think often about a study I performed when I was a fellow at Memorial with Murray Brennan and Marty Karpeh. ${ }^{1}$ I spent many weekends in the basement reviewing paper charts on every patient with a diagnosis of diffuse large B cell gastric lymphoma during a 10-year period. The patients treated with surgery alone had a $100 \%$ cure rate. 


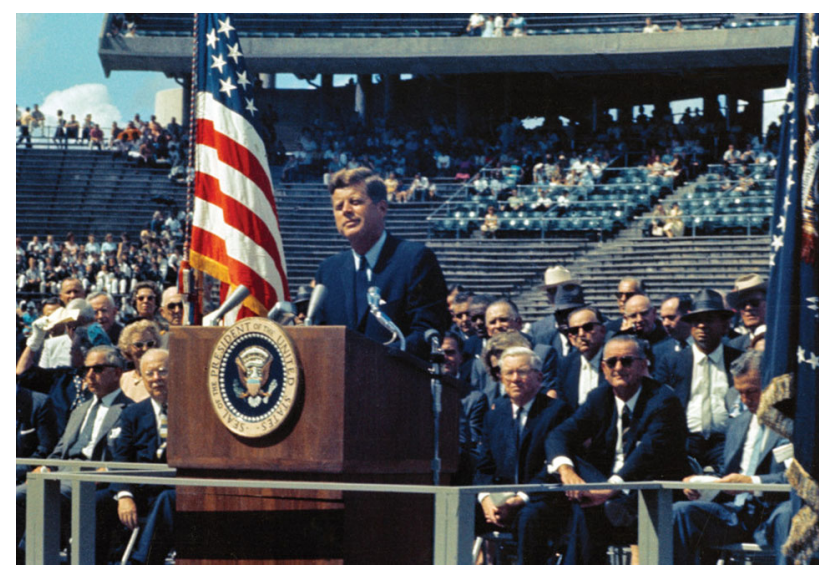

FIG. 1 Photo of President John F. Kennedy announcing the moonshot program at Rice University to the public (courtesy of NASA)

Yet, when was the last time any surgeon operated on gastric lymphoma? It is no longer a surgical disease. Standard therapy for stage 1 or 2 gastric lymphoma is six cycles of R-CHOP followed by $3600 \mathrm{cGy}$ of radiation.

I do not need to explain that a complex chemotherapy regimen for lymphoma can lead to perforations and bleeding, neutropenia, hair loss, and a very poor quality of life for the 5-month duration of therapy, as well as the long-term risks of myelodysplastic syndromes and secondary malignancies. Moreover, not all of these patients are cured.

A minimally invasive distal or even a subtotal gastrectomy can be extremely well tolerated with fewer long-term effects than systemic chemotherapy, but this will never be revisited. Nonoperative management is a goal and measure of success for medical and radiation oncologists. Consider the recent American Society of Clinical Oncology (ASCO) description of the greatest achievements in oncology for 2019, naming "the refinement of surgical treatment of cancer" as the Advance of the Year, recognizing "the effectiveness of novel systemic therapies in reducing the amount of surgery, and even the need for it.". 2

Another example is rectal cancer with its "watch and wait" approach. We all agree that total neoadjuvant chemotherapy and radiation therapy followed by "watch and wait" for complete clinical responders is a great advance for patients who have low, bulky rectal cancer with significant comorbidities otherwise requiring a complicated abdominoperineal resection (APR). But what about mid to high rectal cancers with T3N0 disease? At many institutions, patients with these cancers currently are routinely also treated with total neoadjuvant therapy and "watch and wait." We are seeing the ball roll down a hill, and it is going to be hard to push it back up. A cohort of patients exists that would benefit from neoadjuvant proctectomy with "watch and wait" for systemic chemotherapy. For some patients, a surgery-only approach would be simpler, safer, cheaper, and associated with a better quality of life and long-term survival than the alternative. We need to identify these patients.

A similar path may be followed with breast cancer. Systemic chemotherapy and local stereotactic radiosurgery of breast cancers is advancing and could be seen as an alternative to lumpectomy. Patients should not lose the opportunity to have a safe, curative, non-mutilating surgery for breast cancer. But who except surgeons will study the "who, what, when, and how" behind this goal?

One of my former faculty members once said to me, “There really isn't anything left to do in surgical oncology research. We have learned to be as radical as possible and as minimalist as possible. The only new advances will be in medical oncology." Clearly, he had not read the manuscript by S. Canavero, a neurosurgeon who published the technical details of transplanting the head. ${ }^{3}$ I can imagine a patient going to sleep with widely metastatic cancer devastating the body and waking up with a brand new body. Although I am not advocating for this research, the point is that there is always more that can be explored.

The key to the success of the moonshot program was that America had a far-reaching but achievable goal. We could imagine being successful. Kennedy did not say, "In 10 years, let's better understand cold gas thruster technology as it relates to thrust vectoring and the gravitational well." He also did not say, "In 10 years we will have vacation homes in the Andromeda galaxy." The goal was to land on the moon, and the technology grew around that. In surgical oncology we need to define those practical, surgical goals that will advance all aspects of cancer surgery.

Because I have been thinking extensively about how I can direct my research career to have a meaningful impact on surgical oncology, I share three goals that I think the society and its members should embrace.

The first moonshot goal is to cure $95 \%$ of the patients undergoing surgery for primary cancers. This is not to say that we should expect surgery alone to cure every patient, but that if we are embarking on surgical treatment, the patient should be cured. We have made the right decision to operate. We have managed perioperative factors that have an impact on cancer recurrence, and we have used the optimal adjuvant treatment to address micro-metastatic disease.

I believe that systems biology, artificial intelligence, and machine learning have the potential to help us make decisions about surgery in real time with real-time data. Haroon Choudry and I have a database of more than 2000 patients who have been treated with cytoreductive surgery and hyperthermic intraperitoneal chemotherapy (HIPEC) 
by Drs. Choudry, Holtzman, Ahrendt, Zeh, Pingpank, and myself. Patrick Wagner as a fellow developed a nomogram for making decisions that includes four factors. ${ }^{4}$ This is an example of where the majority of surgical prognostication is stuck. Imagine your financial advisor using a nomogram of four statistically significant predictive factors on multivariate analysis published 3 years ago from data generated more than 10 years earlier to advise you on where to invest your money. Financial advisors use machine learning to take into consideration physical factors, psychological factors, rational and irrational behaviors, day of the week, holidays, time of year, international crisis, pandemics, and more to make predictions. Baseball coaches have more information at their fingertips than surgeons have on predictive outcomes. You cannot separate radiologic tumor stage from the tumor microenvironment, the nutritional status of the patient, the genetic profile of the tumor, the psychological frailty of the patient, the time the tumor has had to grow, the immune microenvironment, the patient's comorbidities, the microbiome, marital status, and family support (Fig. 2). Using artificial intelligence to help us assess all these factors in real time represents our need. As surgeons, we have access to all the information at this point, but we need to use machine-learning technology and artificial intelligence to analyze it and predict the surgical cure, ideally in real time with real-time data obtained directly from electronic medical records.

To cure cancer with surgery, we need to know whether the tumor has already metastasized at the time of surgery. At the moment, for most solid tumors, we rely on radiographic imaging to predict who has metastatic disease. We need better predictive biomarkers. The technology is commercially available to examine circulating tumor cells, tumor DNA, miRNA, exosomes, and even the $\mathrm{T}$ cell repertoire, yet very few papers in the literature have examined these assays in the perioperative setting to predict recurrence after surgery for cancer.

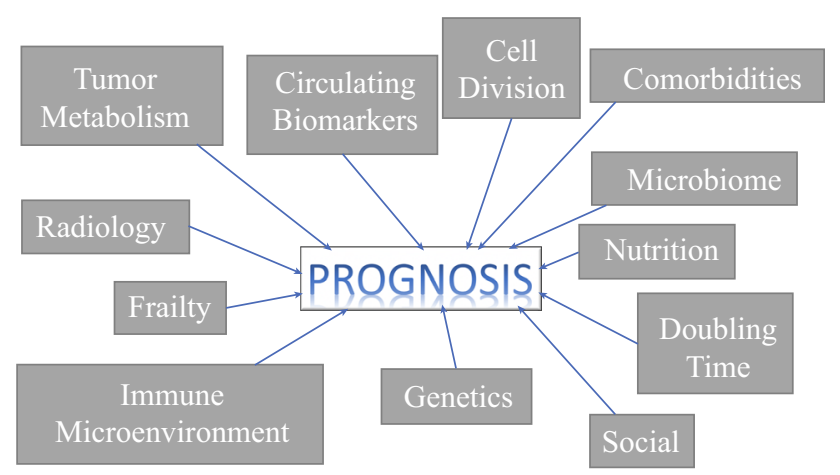

FIG. 2 Schematic showing the complexity of factors influencing prognosis after surgical resection for cancer
As a society, we must ask ourselves whether the ease of presenting and publishing case series is having a negative impact on our field. Young surgeons need to think about practical problems, develop hypotheses, and without being intimidated, use the latest technology to solve them. It takes as much time to publish a case series as it does to analyze serum for biomarkers. We simply need to think about practice-changing research and ask the right questions.

Perioperative stress and complications clearly have an impact on recurrence, and who but surgeons are going to find solutions to this problem. A patient of mine had a slow-growing rectal cancer recurrence for 12 years that finally became symptomatic (Fig. 3). I resected the tumor, but it was complicated by a pelvic infection. The 3-month postoperative computed tomography (CT) scan showed widespread new lung metastases, and the patient died of metastatic disease 9 months after surgery.

According to many of my patients, surgery exposes tumors to air, and air makes cancer grow faster. Their observation may be right. The deleterious effects of surgery include the initiation of local and/or systemic inflammation, increased catecholamine levels, immunosuppression, a prothrombotic state, release of neutrophil extracellular traps (NETs), and damage-associated molecular patterns (DAMPS). These processes overlap with cancer-promoting signaling and metastatic pathways. Cancer cells that escape resection are subject to perioperative physiologic changes and might disseminate and colonize distant organs, thus contributing to postoperative cancer recurrence.

Lorenzo Ferri discussed his research into NETs at the Society of Surgical Oncology (SSO) meeting in 2017. Alan Tsung and Samer Tohme discovered high levels of NETs and high mobility group box 1 (HMGB1) protein in a model of liver cancer after surgery, noting that inhibiting this activity by using deoxyribonuclease (DNAse) can improve cancer-related survival. ${ }^{5}$

As we identify patients who will have recurrence after surgery, we need to design postoperative adjuvant trials that can have an impact on recurrence. Adaptive designs can minimize the number of patients needed and answer questions quickly. Nobody has done this better than Laura Esserman with the I-spy trials for breast cancer. The adaptive design is a unique protocol whereby five new drugs are examined simultaneously and added to standard regimens of neoadjuvant randomized trials for patients with locally advanced breast cancers using complete pathologic response as the end point. Known and exploratory biomarkers are used to stratify and direct accrual. These trials have explored 16 different new agents in the neoadjuvant setting in breast cancer since 2010, and three new drugs have received accelerated approval through that process. 
FIG. 3 Case report of a patient with a 12-year history of local rectal cancer recurrence who succumbed to the rapid development of lung metastases after surgery

\section{Case Report: Rectal Cancer}

- November 2005

- Low Anterior Resection T3N1

- Adjuvant Chemotherapy

- Radiation therapy

- July 2017

- Hematuria

- Local Recurrence diagnosed by cystoscopy and scan

- August 2017

- Pelvic Exenteration

- Complicated by pelvic abscess and sepsis, 20 day hospital stay

- October 2017

- 25 new lung metastases

- May 2018

- Succumbed to lung metastases
Therefore, getting to a 95\% cure means we must address many obstacles such as prognosticating better through systems biology and machine learning, improving biomarkers, examining the tumor microenvironment (TME), inhibiting negative perioperative factors, and improving adjuvant therapy (Fig. 4). Altogether, as we accomplish this goal we will have advanced essential technology on many fronts.

\section{Goal 1: 95\% Surgical Cure}

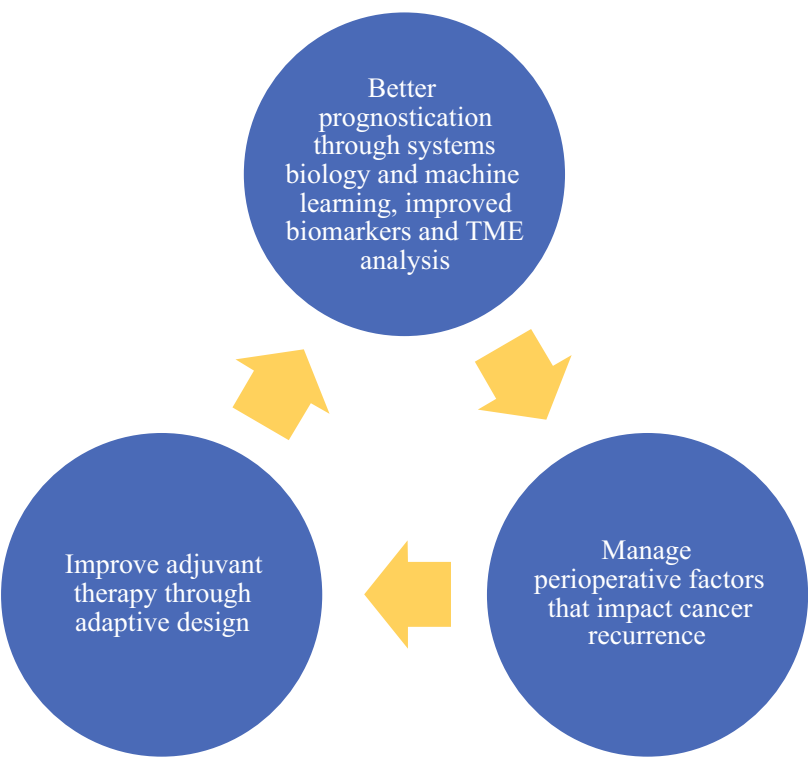

FIG. 4 Interconnecting research opportunities that must be addressed as part of the surgical oncology moonshot goal of a $95 \%$ surgical cure
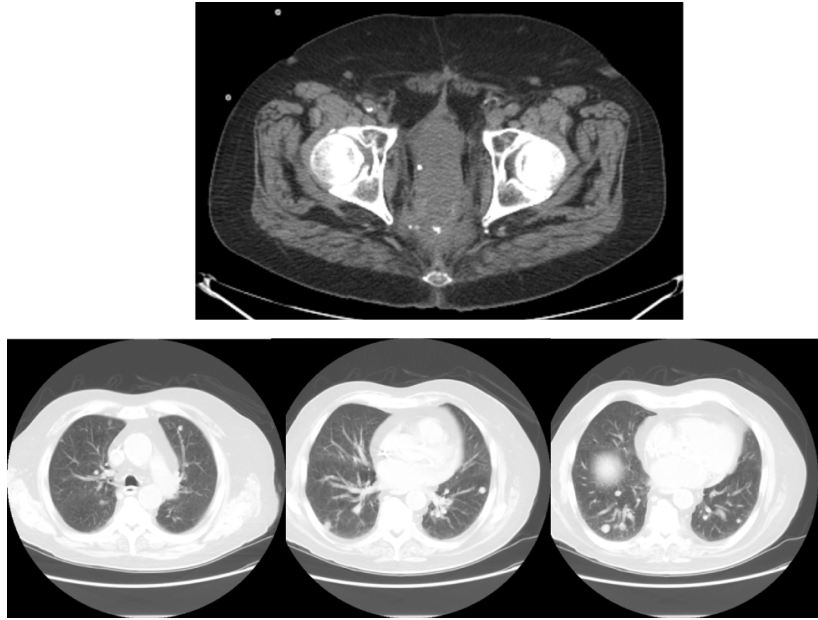

The second moonshot goal is to minimize the impact of cancer surgery such that no oncologic operation results in a hospital stay longer than $48 \mathrm{~h}$. That may seem unfeasible and unnecessary, but I argue that it is essential to the future of our specialty.

Cholecystectomies are same-day surgeries. When I started my training, a cholecystectomy resulted in a 7-day hospital stay with a month off work. The risk of wound complications and drains had a major quality-of-life impact. If we as surgeons had not improved the impact of our surgery, all of these patients would be managed with a percutaneous drain and antibiotics, or gastroenterologists would have figured out a way to take the gallbladder out without our help. Hip replacement surgery is a same-day surgery. Patients can have their aortic valve replaced and be home in 2 days. Patients with gastric bypass surgery and two anastomoses go home within $24 \mathrm{~h}$ with less than a $1 \%$ mortality risk.

In general, surgical oncology has been behind the curve when it comes to optimizing surgical technique and perioperative management, but I think our slow adoption of new surgical and perioperative methods is a mistake. Suzanne Klimberg, Charles Scoggins, and I started the SSO Fellows Institute to show the next generation of surgical oncologists that SSO embraces technology. I became interested in robotics after visiting Dr. Pigazzi at the City of Hope and participating in a minimally invasive surgical oncology conference in 2007. The robotic technology highlighted at that conference offered significant advances beyond straight laparoscopy for intracorporeal suturing. Soon thereafter, Sri Chalikonda and I visited Pier Giulianotti to watch his technique for robotic Whipple, and within a few weeks Herb Zeh, Sri, and I performed the first robotic Whipple at the University of Pittsburgh. Herb Zeh and Jim Moser then developed a streamlined approach to 
the robotic Whipple and rigorously studied the technique without the bias that it was better than the standard approach. At Pitt, we had performed more than 500 robotic Whipple operations and had recently reported those results. Thanks to Melissa Hogg, who developed a robust training program, we have trained 25 fellows and too numerous to count attending surgeons across the world to perform this technique.

So how close are we to a 48-h stay after Whipple? A patient of Amer Zureikat's at the University of Pittsburgh presented with obstructive jaundice, and the workup showed a pancreatic head mass. The patient received neoadjuvant chemotherapy on trial, then underwent a robotic Whipple as well as an enhanced-recovery-aftersurgery (ERAS) protocol for perioperative management. The drain was removed on postoperative day (POD) 2, and the patient was discharged on POD 4 with no readmission, then resumed chemotherapy within 30 days.

Clearly, the 48-h hospital stay after Whipple is not only about the robotic approach. It is about patient education, pre-habilitation, algorithm-based perioperative care, ERAS protocols, enhanced home care, non-narcotic pain control, and avoidance of unnecessary tubes and drains (Fig. 5). All that we learn in accomplishing this goal will apply to other complex surgical oncology procedures. This will improve the acceptance of surgery, improve the cancer-related outcome, and enhance the ability to use surgery as part of a multidisciplinary program, with patients adjusting to their adjuvant therapy without experiencing undue debilitation.

The final moonshot goal is to prevent patients from dying of peritoneal or liver metastases. We need to continue exploring innovative ways to use surgical techniques other than resection alone to treat regionally advanced cancers (Fig. 6). This is what I have dedicated much of my clinical career to doing. I have focused on using surgery to

Goal 2: $<48$-hour hospital stay for all Cancer Surgery

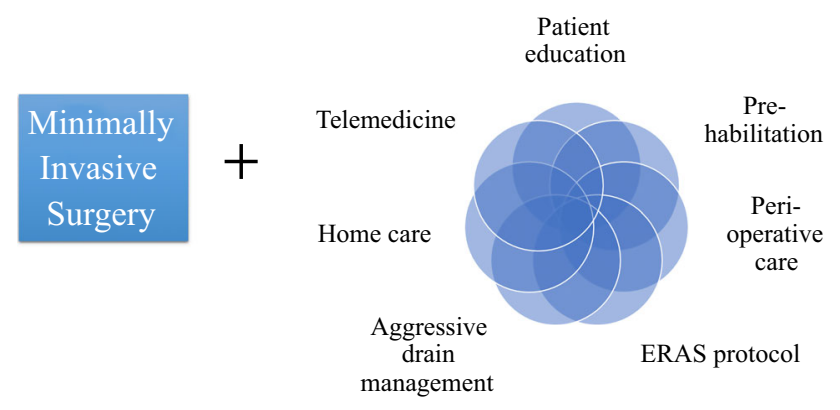

FIG. 5 Combined improvements in perioperative management that must be studied and refined to achieve the surgical oncology moonshot goal of a hospital stay shorter than $48 \mathrm{~h}$ for all cancer surgery deliver biologic agents and chemotherapy to regions of the body for cancers beyond standard resection options yet still regionally confined.

This program, initiated at the National Cancer Institute (NCI) by Doug Fraker and Rich Alexander and advanced by myself and Steve Libutti, has included regional perfusions for the limb, the liver, the peritoneum, the pleura, the lung, and I think even the kidney at one point. This still is a unique avenue of research for surgical oncologists to expand their tools in a way to help cancer patients. My goal when I left the NCI was to prove that these programs could be developed outside the walls of the National Institutes of Health (NIH) clinical center and to develop this as a field of collaborative research in centers across the United States.

The majority of academic surgical oncology programs currently have programs in regional therapy, centered primarily on peritoneal metastases. Credit is given to Paul Sugarbaker for this field. Rich Alexander, Clark Gamblin, and I started the Regional Therapies Conference for the sharing of research and clinical experience around regional therapies. This grew from a handful of surgeons presenting their research in a small room in Pittsburgh to a strong group of more than 200 surgeons and more than 125 abstracts presented at Orlando in 2020. The SSO has taken this meeting on, renaming it "Advanced Cancer Therapies" and demonstrating the commitment to education and research around advanced surgical therapies that extend the use of surgical techniques for advanced cancers (Fig. 7). The goal is to share ideas and data that expand the role of surgery beyond classically resectable tumors. I look forward to SSO's continuing development of this opportunity to address this essential need of our field and encourage the consideration of additional similar conferences to focus on minimal-impact surgery as well as biomarkers and perioperative interventions.

My laboratory has been dedicated to an exploration of regional immunotherapy using tumor-selective oncolytic vaccinia virus expressing novel immunogens. I anticipate moving forward with a clinical trial of intraperitoneal oncolytic vaccinia virus expressing a re-designed interleukin (IL)-2 into a clinical trial involving patients with peritoneal metastases from colon cancer. We have found that it is uniquely effective and safe in animal models, and as with every immunotherapy approach, it works better in combination with checkpoint inhibitors. I know that intraperitoneal and intrahepatic chimeric antigen receptor $\mathrm{T}$ (CAR-T) cells and novel nanoparticles for sustained intraperitoneal release of cytotoxic drugs are being explored in clinical trials. Imagine the right clinical trial network with the appropriate design and end point that allows a series of trials such as the I-spy trials enabling us to know what combination of new regionally applied drugs would solve unresectable liver metastases or peritoneal 
FIG. 6 Examples of regional therapy approaches needing research efforts to address the surgical oncology moonshot goal to eliminate peritoneal and liver metastases as the cause of patient deaths
FIG. 7 The Society of Surgical Oncology (SSO) Advanced Cancer Therapies meeting was developed to encourage the presentation of research about expanding the mission of surgical oncologists beyond standard resections for cancer

\section{Goal \# 3. No Patient Should Die from Peritoneal or Liver Metastases}

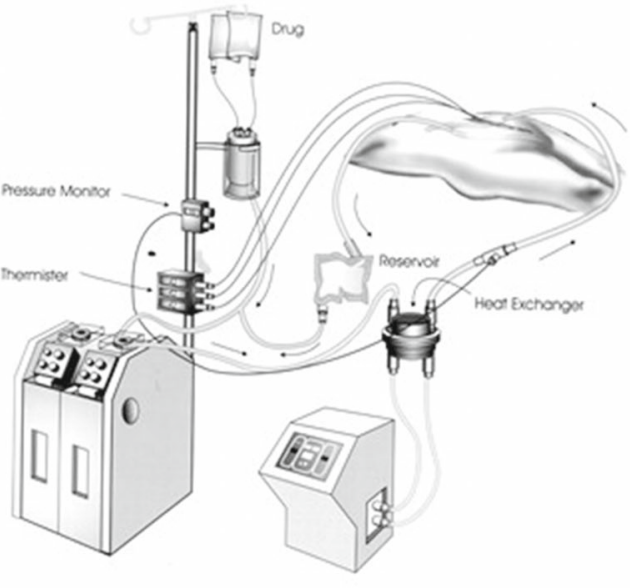

Heated Intraperitoneal Chemoperfusion

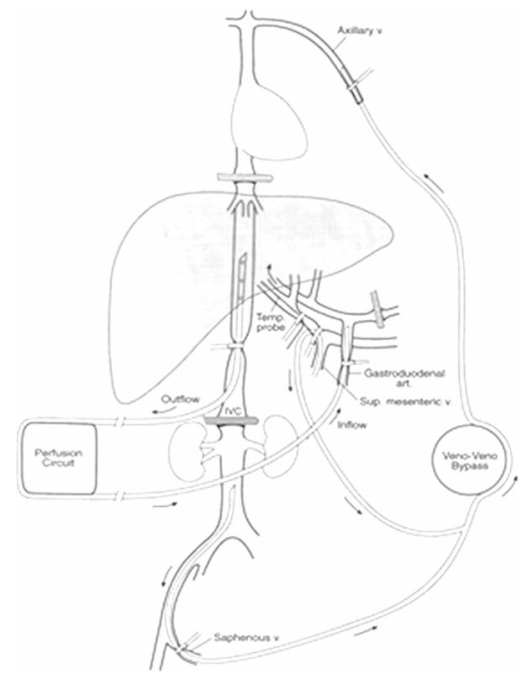

Isolated Hepatic Perfusion

Advanced Cancer Therapies:

Expanding the Mission of Surgical Oncologists Beyond Standard Resections

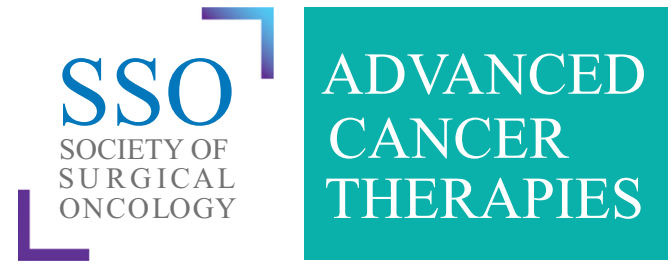

Formerly known as The International Symposium on Regional Cancer Therapies

\section{Abstracts Submitted \& Presented}

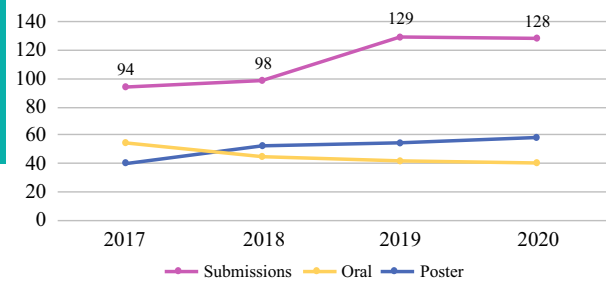

metastases. We need a new hero to step up and help move this approach forward. Charles Staley is trying to make this happen through the Eastern Cooperative Oncology Group (ECOG) and deserves all our support.

Surgeons used to lead the charge for cancer research. I had the honor of working with Bernie Fisher during the last 20 years at the University of Pittsburgh. Bernie Fisher passed away this year at the age of 101 years, after a 75-year career leading oncology research (Fig. 8). At the same time that Sputnik was flying in 1957, he was helping to establish the National Surgical Adjuvant Breast and Bowel Project (NSABP) at the direction of I. S. Ravdin, the chair of surgery at the University of Pennsylvania. Fisher had the determination and fortitude to rally the surgical community around enrolling patients in randomized clinical trials to test hypotheses regarding the biology of cancer. This included all surgeons in both academic and community programs. He was responsible for defining the paradigm of how cancers metastasize and transforming the way the world thought about cancer and its treatment. He was the world leader in clinical oncology research.

In addition, others have led the way in clinical oncology research: Steve Rosenberg, Murray Brennan, Charles Balch, Don Morton, Armando Giuliano, Norm Wolmark, Sam Wells, Heidi Nelson, Monica Bertagnolli, Suzanne Topalian, Kelly McMasters, Ron Dematteo, Laura Esserman, and Monica Morrow all ran pivotal trials. Among these idols are chairs of surgery; presidents of SSO and ASCO; Lasker award winners; and Nobel candidates. The accolades and titles came after these leaders published their clinical trials, so the road to academic success and career fulfillment is through a randomized clinical trial-a simple formula.

Why then has the culture of surgical oncology research changed and what can we do about it? Is it because we are lost and intimidated when it comes to the enormous technical advances in research tools? Is it because of bias 
FIG. 8 Bernard Fisher was a surgeon scientist who led the world in clinical trials for cancer, and his legacy challenges surgical oncologists to again take on the charge of practice-changing clinical research
"I looked upon clinical trials as an extension of my laboratory, and I appreciated that they could be used as a methodology for testing hypotheses. Until their use anecdotalism was the primary source of information for determining treatment strategies. Consequently, using clinical trials to evaluate our alternative hypothesis seemed appropriate,"

Journal of the National Cancer Institute, Vol. 97, No. 22, November 16, 2005

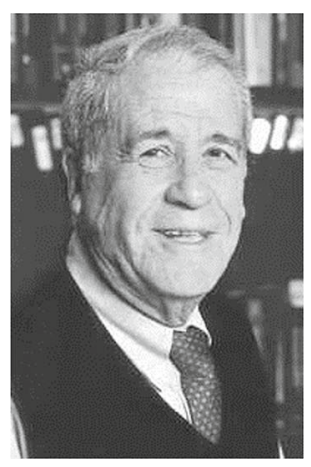

August 23, 1918 - October 16, 2019

against surgeons for research funding? Is it because we are beholden to relative value unit (RVU) counters and cannot find the time? The answer is yes to all. We lack knowledge, money, and time. Yet all these can be overcome if we have passion and a goal. Howard Thurman, an author and philosopher, once said "Don't ask yourself what the world needs; ask yourself what makes you come alive. And then go and do that because what the world needs is people who have come alive."

I hope you can be passionate around these goals or other moonshot goals the society puts forth. With passion, we will understand how transcriptomics and artificial intelligence can tell us which patients to treat with surgery. With vision, we will find funding through grateful patients, industry, hospital support, and the NIH, and with fortitude, we will spend the extra hour or two a day after long operations to make progress. We need to work as a coordinated team, and the SSO should help organize that team.

The real moonshot required practical solutions to the goal of putting a person on the moon. It was in no way ignorant of new technology and an understanding of new physics, but imagine if Kennedy had put the physicists in charge of putting a man on the moon. My father was a mechanical engineer working in the aerospace industry, and he used to describe to me the difference between physicists and engineers. He said, "Physicists study the world as it is; engineers create the world that has never been." Surgeons can create a world that has never been.

I will finish with an allegory. Surgical oncologists have a unique skill set for tackling practical problems in oncology. History has many examples of complex problems solved by practical people with practical solutions. The Gordian knot is one of these. In 2000 BC, King Midas tied an oxcart to a post with a number of intricate knots.
The knot survived for 1700 years, at which point an oracle decreed that anyone who could untie the knot would be the ruler of all Asia. As might be imagined, molecular biologists, physicists, genomics experts, and immunologists all studied the complex knot microenvironment for 100 years, leading to numerous oral abstract presentations, high-impact manuscripts, grants, tenure appointments, endowed professorships, and research awards, but the cart did not move. Then along came Alexander the Great, who pulled out his scalpel (or sword) and sliced the knot in half, allowing it to unravel (Fig. 9).

Surgeons have an essential role in cancer treatment and research, and we cannot let that slip away. Although I am a supporter of the academic surgery model, this surgical oncology moonshot is not the realm only of traditional academic institutions. I have taken on the exciting role of building the most productive translational research platform in the country as Cancer Institute Chair at Allegheny Health Network in Pittsburgh. We will be studying practical solutions to the biggest problems in cancer care while leveraging the integrated delivery and finance system (IDFS) model, corporate engagement, entrepreneurism, philanthropy, and collaboration with forward-thinking companies and institutions. After all, Apple, Google, and Amazon have transformed the world in goal-oriented ways that could not have been predicted by traditional academic institutions. All SSO members should participate in this surgical oncology moonshot, setting the bar high, changing the culture by moving surgeons from the bottom of the research hierarchy to the top, and solving difficult problems in practical ways.

It has been an honor to serve as president this year, and I thank the other leaders with whom I have worked closely including the executive committee, the executive council, 
FIG. 9 Fedele Fischetti's (Naples 1732-1792) painting of Alexander cutting the Gordian knot, oil on canvas

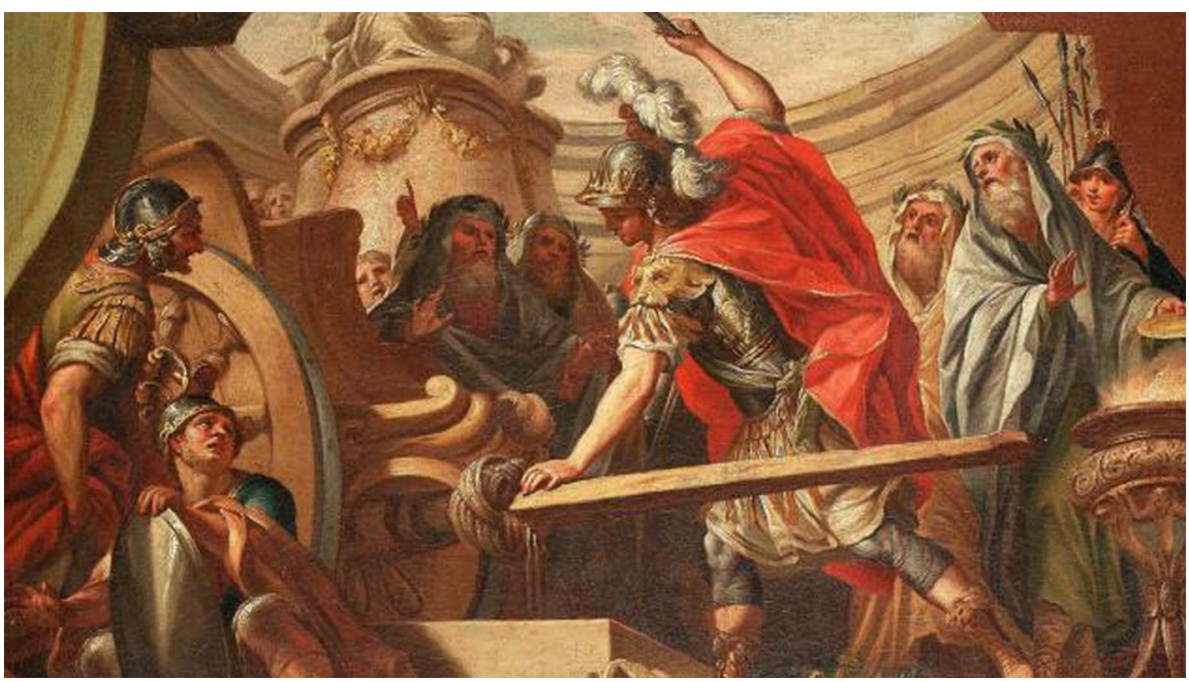

FIG. 10 a Chad, Chrissy, Susan, and David Bartlett on vacation in the Bahamas, 2018. b Susan and David at the Southern Surgical Meeting, 2019
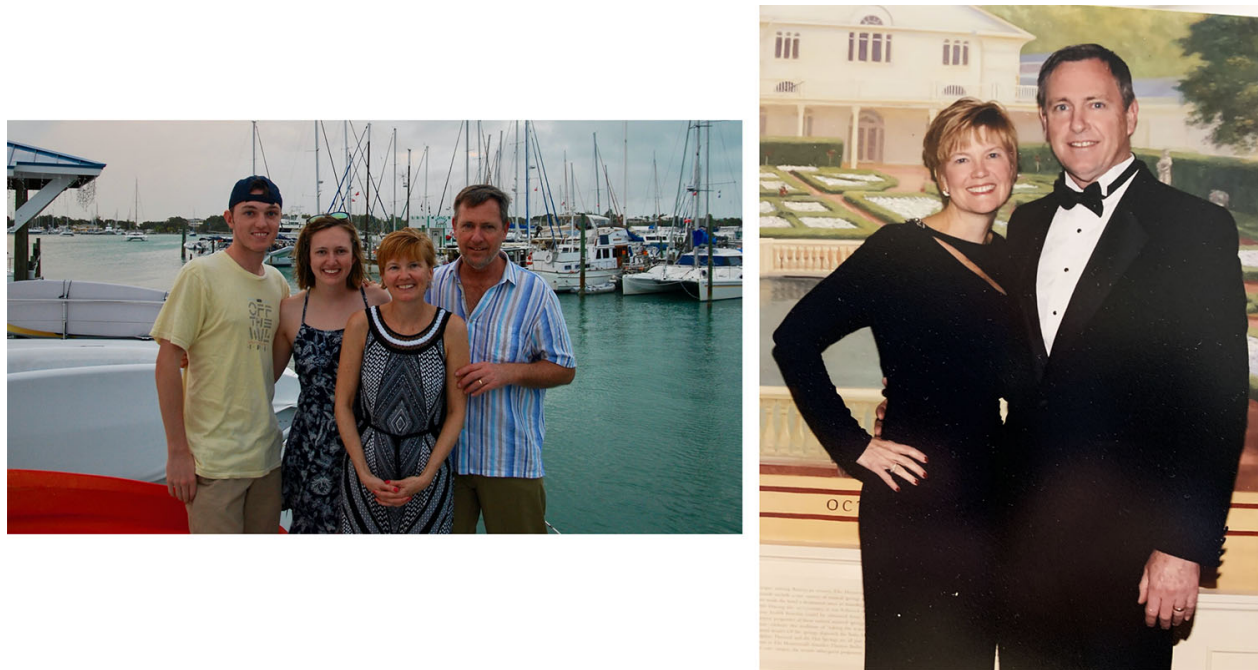

and all the hundreds of volunteers who make this organization hum. I especially thank the staff of SSO. Eileen Widmer and her outstanding team have stayed on top of everything throughout this difficult time, and it was all I could do to keep up with them.

I am in debt to my mentors: John Daly, Murray Brennan, Steve Rosenberg, Dan Coit, Rich Alexander, Charles Balch, Tim Billiar, Mike Lotze, Bernie Fisher, and many others. I publicly acknowledge those who have intentionally or surreptitiously helped my career: Jeff Drebin, Mitch Posner, James Economou, Suzanne Klimberg, Marty Heslin, Mike Chen, and so many others. I was fortunate to have outstanding partners at NCI, Pitt, and now AHN: I especially recognize Herb Zeh, who joined me at Pitt at the beginning with a similar work ethic and passion for building a program. Herb was a partner, friend, confidant, and colleague for 17 years.
I am particularly grateful to my loyal staff: Heather Jones, Margaret Corson, Maureen Hewko, and Sue Schoonover, and so many others. I am most proud of all of our current and former trainees throughout the country. They have been my inspiration, and their camaraderie and loyalty to our program, our faculty, and each other have been remarkable.

Finally, I thank my family (Fig. 10). As with many of you, my family had to sacrifice for my aspirations. My kids grew up never having breakfast, lunch, or dinner with me on a weekday. Think about what that means. Not being responsible for their table manners is a small example of how I was not around enough to raise them, not around enough to celebrate important events in their lives, and not around enough to help them through difficult times. I was simply not around enough. Yet thanks to my wife Susan, they turned out great, and I could not be more proud of who 
they are. I did, however, manage to have enough time to play games and sports with them, which I cherish and continue to treasure more than they will ever comprehend.

Finally, Susan, in addition her role as an exceptional nurse practitioner in our surgical oncology practice and a partner on our team, has been as devoted to my career as I am. It is humbling, and something I can never pay back. As I get older, I realize that the most important thing we can accomplish on this earth is a lasting, loving relationship, and if that were as easy as walking on the moon, the world would be a utopian dream. Somehow, Susan and I have had that for 30 years and counting. I am lucky, and I thank her.

\section{REFERENCES}

1. Bartlett DL, Karpeh MS Jr, Filippa DA, Brennan MF. Long-term follow-up after curative surgery for early gastric lymphoma. Ann Surg. 1996;223:53-62. https://doi.org/10.1097/00000658-199601 000-00008.
2. Markham MJ, Wachter K, Agarwal N, et al. Clinical cancer advances 2020: annual report on progress against cancer from the American Society of Clinical Oncology [published correction appears in $J$ Clin Oncol. 2020;38:3076. J Clin Oncol. 2020;38:1081. https://doi.org/10.1200/jco.19.03141.

3. Ren X, Li M, Zhao X, et al. First cephalosomatic anastomosis in a human model. Surg Neurol Int. 2017;8:276. https://doi.org/10.41 03/sni.sni_415_17.

4. Wagner PL, Austin F, Zenati M, et al. Oncologic risk stratification following cytoreductive surgery and hyperthermic intraperitoneal chemotherapy for appendiceal carcinomatosis. Ann Surg Oncol. 2016;23:1587-93. https://doi.org/10.1245/s10434-015-5037-7.

5. Tohme S, Yazdani HO, Al-Khafaji AB, et al. Neutrophil extracellular traps promote the development and progression of liver metastases after surgical stress. Cancer Res. 2016;76:1367-80. https://doi.org/10.1158/0008-5472.can-151591.

Publisher's Note Springer Nature remains neutral with regard to jurisdictional claims in published maps and institutional affiliations. 